\title{
Interroger le paradigme conradien
}

Jean-Pierre Orban et Maëline Le Lay

\section{OpenEdition \\ Journals}

Édition électronique

URL : https://journals.openedition.org/coma/2961

DOI : $10.4000 /$ coma.2961

ISSN : 2275-1742

\section{Éditeur}

Institut des textes \& manuscrits modernes (ITEM)

\section{Référence électronique}

Jean-Pierre Orban et Maëline Le Lay, «Interroger le paradigme conradien », Continents manuscrits [En ligne], 11 | 2018, mis en ligne le 15 octobre 2018, consulté le 13 janvier 2023. URL : http:// journals.openedition.org/coma/2961 ; DOI : https://doi.org/10.4000/coma.2961

Ce document a été généré automatiquement le 13 janvier 2023.

\section{(c) (i) ()}

Creative Commons - Attribution - Pas d'Utilisation Commerciale - Pas de Modification 4.0 International - CC BY-NC-ND 4.0

https://creativecommons.org/licenses/by-nc-nd/4.0/ 


\section{Interroger le paradigme conradien}

Jean-Pierre Orban et Maëline Le Lay

\section{La « matière » Congo infiltrée par le filon conradien}

Ce dossier fait suite à une demi-journée d'étude organisée au Musée du Quai Branly le 9 juin 2018.

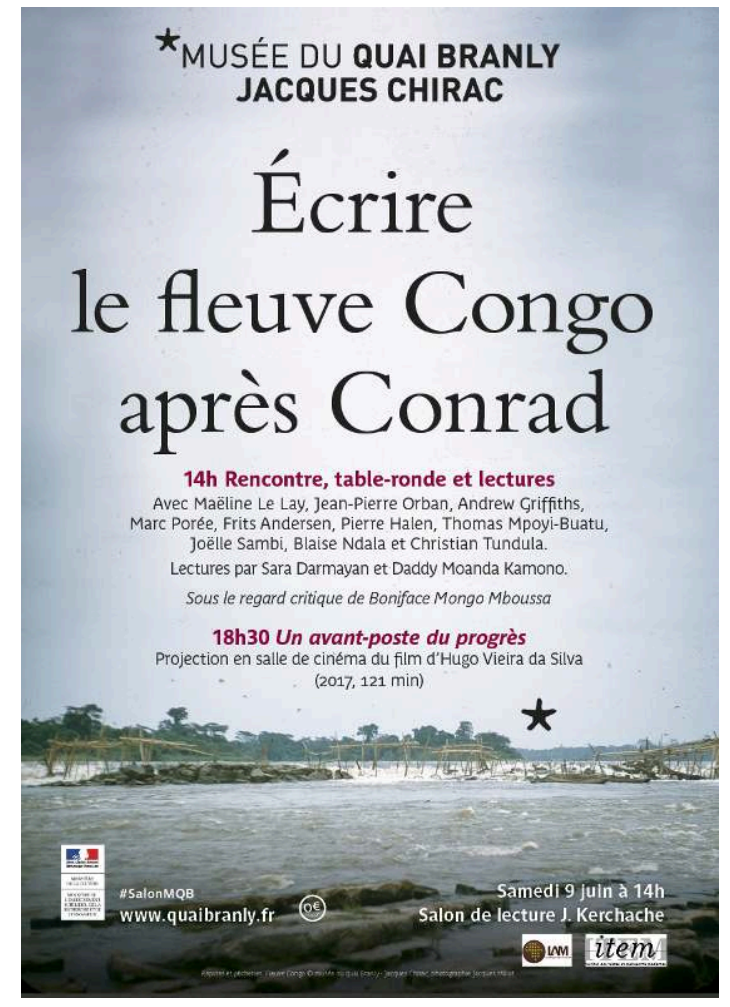

2 Nous avions souhaité y réunir écrivains, artistes et chercheurs, tou.te.s concerné.e.s, à différents égards et sous des formes variées, par Joseph Conrad et sa célèbre novella, $\mathrm{Au}$ cœur des ténèbres ${ }^{1}$. Il s'agissait de discuter ensemble de l'existence d'une supposée préemption du « filon » conradien dans la « matière » $\mathrm{Congo}^{2}$. 
3 En effet, dès qu'il s'agit, pour les Occidentaux, d'écrire sur le Congo, surgissent presque systématiquement des références au « cœur des ténèbres ». La novella de Conrad semble avoir comme imprimé sa marque à la littérature sur le Congo - récits de voyage, romans et écrits journalistiques - au niveau du champ sémantique auxquels se réfèrent les auteurs de ces textes comme de l'atmosphère qui s'en dégage. Comme si c'était dans ce bref et dense roman que se constituait la trame généalogique originelle, le substrat de base (la "matière" Congo) pour parler du Congo, le Congo Kinshasa/RDC essentiellement, mais aussi le Congo Brazzaville, la géographie de ce que recouvre ce nom étant à géométrie variable, des frontières nationales au Royaume Kongo $^{3}$ en passant par le Bassin du Congo qui irrigue plus largement l'Afrique centrale.

Le Congo est-il indissociablement lié, rivé presque, à Joseph Conrad, et à sa novella $\mathrm{Au}$ cœur des ténèbres? Comment le paradigme conradien en est-il arrivé à informer aussi profondément la littérature européenne du $\mathrm{xx}^{\mathrm{e}}$ et du $\mathrm{xxI}^{\mathrm{e}}$ siècles et, au-delà, le discours médiatique et, au-delà encore, le discours populaire? Par "paradigme conradien ", nous entendons le modèle de représentations qu'a constitué - à partir de la tâche que Conrad s'assignait, avant tout, de faire voir ${ }^{4}$ en une démarche simili-picturale - le court récit «africain » de Conrad dans l'imaginaire collectif, mais aussi ce qui l'a nourri et suivi de près en le renforçant: toute une généalogie de textes littéraires, journalistiques et administratifs sur l'exploration du Congo, puis son exploitation dans l'État Indépendant du Congo (1885-1908) sous la souveraineté du roi des Belges, Léopold II. Une généalogie à laquelle appartiennent les récits de voyage d'H.M. Stanley ${ }^{5}$, le rapport de Roger Casement ${ }^{6}$ sur les " atrocités » du régime léopoldien (1903), la satire King Leopold's Soliloquy ${ }^{7}$ de Mark Twain (1905) ou The Crime of the Congo ${ }^{8}$ de Conan Doyle (1909).

5 Ce sont ce paradigme et cette généalogie qui contiennent et produisent les connotations pour longtemps associées au territoire et au fleuve qui le parcourt comme une épine dorsale. Des connotations qui, d'obscurité physique (celle, réelle et imaginaire, de la forêt équatoriale) et du primitivisme racial, passent rapidement aux ténèbres morales et à la sauvagerie d'une humanité douteuse ${ }^{9}$ en un retour aux premiers temps oubliés de l'Histoire ${ }^{10}$ (" nous étions des voyageurs sur la terre de la préhistoire... »).

6 En 1878, Henry Morton Stanley publie Through the Dark Continent ${ }^{11}$. En 1890, il fait paraître In Darkest Africa, un ouvrage qui sera traduit la même année en français sous le titre Dans les ténèbres de l'Afrique. 1890, c'est aussi l'année où Conrad effectue son voyage au Congo. Neuf ans plus tard, il publie Heart of Darkness en magazine, un récit empreint de celui de son prédécesseur à la manière d'un palimpseste. Les échos avec les textes de Stanley témoignent en effet d'une troublante intertextualité dont nous entretient Andrew Griffiths («Seeking the sources of Heart of Darkness: the African narratives of late-Victorian Explorer and Journalists »).

7 En substantifiant le mot « dark » en " darkness », Conrad, volontairement ou non, fait de cette obscurité un état de fait, comme on dessine un lieu. De la même manière, avec le titre $A u$ cour des ténèbres, s'ajoute imperceptiblement en français la dimension morale et même religieuse associée au mot «ténèbres » : l'enfer et la faute qui vous y jette.

8 Sachant que ce ne sont pas tant les récits de Stanley qui imprégneront l'inconscient collectif dans le monde francophone où les œuvres de l'explorateur, et même sa vie, sont très étrangement assez peu connues, c'est la novella de Conrad - et, plus encore, 
son titre - qui frappera comme au fer rouge l'esprit francophone, belge et français. S'est ainsi opéré un glissement entre une narration empreinte, chez Stanley, du manichéisme de l'époque, mais ayant pour visée - surfaite - de constitution de « savoirs réalistes ${ }^{12}$ » et une héroïsation de l'explorateur blanc, vers une sélection des passages exotisants, ainsi que le décrit Pierre Halen dans sa contribution («À propos de la traduction conradienne du cœur des ténèbres comme archive internationale »), enfin vers un récit métaphorique où chacun, Blanc ou Noir, comme damné, se perd dans les ténèbres à la fois naturels et éthiques. Mais en un renversement significatif de l'importance des images, ce sont celles-ci qui perdurent et sont prises pour réalités. Comme pour donner raison à Conrad - sans doute, dans un autre sens que celui qu'il imaginait - lui qui écrivait, dans la «note de l'auteur » accompagnant l'édition en recueil d'Au cour des ténèbres:

“Au cœur des ténèbres" est de l'expérience aussi, mais c'est de l'expérience poussée un petit peu (juste un petit peu) au-delà des faits réels de l'affaire, dans l'intention parfaitement légitime, je crois, de la rendre sensible à l'esprit et au cœur des lecteurs. Il ne s'agissait plus là d'une sincérité de coloris. C'était comme un art entièrement différent. Il fallait donner à ce thème sinistre une sombre résonance, une tonalité spécifique, une vibration continue qui, je l'espérais, flotterait dans l'air et se prolongerait dans l'oreille après l'accord final ${ }^{13}$.

9 Cette résonance conradienne aura duré longtemps. Les textes romanesques issus de ce que l'on nomme ici paradigme et que Nicolas Martin-Granel désigne par l'expression " geste conradienne ${ }^{14}$ » se retrouvent jusqu'à aujourd'hui. S'agissant uniquement de la littérature française, on citera Patrick Besson (Mais le Fleuve tua l'homme blanc, 2009), Patrick Deville (Equatoria, 2009), Jean-Christophe Grangé (Lontano, 2015 et Congo Requiem, 2016) et Éric Vuillard (Congo, récit, 2012). Et quand ce n'est pas pour suivre ou réadapter le modèle, c'est le détourner ou se situer par rapport à lui, mais avec l'imprégnation des mêmes représentations : on citera, dans ce sens, Il faut beaucoup aimer les hommes, de Marie Darrieussecq (P.O.L., 2013) ou le récent Tiens ferme ta couronne de Yannick Haenel ${ }^{15}$ (Gallimard, 2017) qui propose un nouveau brassage des thèmes conradiens, de manière décentrée : on y voit passer Conrad et Au cour des ténèbres, mais aussi ses adaptations cinématographiques, Apocalypse Now, film avec lequel le narrateur entretient un rapport obsessionnel. Et à défaut de nous livrer ici à une revue bibliographique exhaustive qui reprendrait également la masse des publications non francophones, on ne citera que deux récits de voyage: Michela Wrong, In the Footsteps of M. Kurtz (Fourth Estate, 2000) et Tim Butcher, Blood River (Chatto \& Windus, 2007) ${ }^{16}$.

\section{Au cœur des ténèbres, une affaire européenne?}

10 Cette apparente omniprésence du paradigme conradien dans la littérature sur - dans un effet de contre-zoom - le Congo, l'Afrique centrale, voire le continent dans sa globalité, nous conduit à formuler une double hypothèse.

11 La réception d'Au cœur des ténèbres ne serait-elle pas qu'une affaire européenne, véhiculant le regard des Blancs sur l'Afrique, notamment sur l'Afrique centrale et son cœur, le Congo, la RDC? Dans un premier temps, ce regard se serait résumé à une vision de l'enfer et de la barbarie dont on aurait eu à extraire les indigènes. Dans un deuxième temps, ce regard serait devenu celui que l'on porte sur les générations de 
colonisateurs, "explorateurs/exploiteurs » et sur l'héritage moral qu'elles ont laissé. Au couur des ténèbres serait alors l'objet et l'expression d'une culpabilité.

Gardons toutefois à l'esprit le fait que cette hypothèse découle d'une lecture postcoloniale qui s'impose comme naturellement à nous aujourd'hui, mais qui ne correspond pas exactement à la réception de Conrad à son époque, comme nous le rappelle Pierre Halen qui fait de l'auteur le tenant d'une tradition d'«exotisme critique »:

It is exactly this conception of "Progress", at once Promethean and humanitarian, that the Conradian tradition, also known as critical exoticism, opposes. It consists less of firsthand accounts of colonial and postcolonial Africa than to a literature devoting in illustrating the failure or unfeasibility of both humanism and positivist materialism. In this respect the tradition could also be described as "radical exoticism", insofar as we seen it, everything is a concept of Otherness - not Victor Segalen's Diverse, much less the Other as it is conceptualized in the discourse of "respect of differences" or "dialogue between cultures"

Aussi est-il loisible de se demander si la lecture d'Au cœur des ténèbres n'a pas été dès le départ biaisée, comme le postule Frits Andersen selon lequel la figure d'un Conrad grand témoin des «atrocités congolaises» est une construction postcoloniale postérieure à la parution d'Au coeur des ténèbres ${ }^{18}$. Et si par la force des choses, de l'Histoire, celle de la domination coloniale, cette lecture n'a pas pesé sur le destin de l'Afrique centrale, du Congo plus précisément, partant sur l'inconscient collectif des Congolais, imposant, dès lors, à ceux-ci, une réalisation du récit : « La parole construit le pays ", ainsi que le dit un proverbe bambara, cité par le personnage de Kena dans $L a R e-$ Production de Thomas Mpoyi-Buatu ${ }^{19}$.

14 À partir de cette première hypothèse, on peut légitimement s'interroger sur la question de savoir si le paradigme conradien informe également la littérature congolaise, plus largement, les arts. C'est cette question générale qui a présidé à l'organisation de la journée d'études du 9 juin 2018 au Quai Branly et dont on peut lire ci-après les articles qui en ont été tirés. Notre hypothèse est que ce paradigme trouve un écho différent dans les créations artistiques congolaises, peut-être à travers une lecture plus critique, ou simplement de manière moins récurrente voire obsédante, que nombre de textes écrits par des Européens marqués par une certaine traduction de l'exotisme artistique dès lors qu'il est question de représenter l'Autre.

Les interventions des écrivains et artistes congolais invités semblent confirmer l'hypothèse : la lecture de la novella de Conrad intervient souvent assez tard dans leur parcours, à la suite d'une incitation extérieure, presqu'incidemment (voir l'intervention de Joëlle Sambi, "Hors Conrad: Congo Eza»). Toutefois, une fois découvert le texte et son impact, les écrivains et les artistes n'en demeurent pas moins poreux à sa rhétorique - même superficiellement - et peuvent opportunément la reprendre à leur compte, par exemple pour ironiser sur son usage inflationniste à tendance sensationnaliste, comme le fait Blaise Ndala dans son roman Sans capote $n i$ kalachnikov. Partant du constat selon lequel l'endroit le plus sombre du Congo (présenté comme tel du moins), l'est du pays, est celui qui, fréquemment, écope aujourd'hui du label conradien de cœur des ténèbres, Blaise Ndala ancre l'intrigue de son deuxième roman au Rwenzori ${ }^{20}$ et dans « la capitale mondiale du viol» (triste sobriquet de la ville de Goma, chef-lieu de la province du Nord-Kivu ${ }^{21}$ ) (Blaise Ndala, «Au cœur des ténèbres, une œuvre ambiguë »). Enfin, Christian Tundula se le réapproprie comme véhicule d'un récit personnel et collectif qui n'a plus rien à voir - hormis la présence du fleuve et les 
drames qui s'y déroulent - avec le contenu original (voir Christian Tundula, « Heart of Darkness, exposition »).

Ainsi ce qui subsiste, c'est la référence au fleuve Congo parcouru jadis par Conrad, demeuré un référent identitaire puissant dans l'imaginaire congolais et repris par les artistes et écrivains sous une tonalité plus urbaine et dès lors moderne, comme en témoigne le portfolio de photographies et de textes (Sony Labou Tansi, Tchicaya U'Tamsi, Henri Lopes, Fiston Mwanza Mujila, Sinzo Aanza, Emmanuel Dongala ou Dieudonné Niangouna) choisis par Nicolas Martin-Granel (« Le fleuve commence ici »).

\section{Au cœur des ténèbres, un malentendu}

17 Au fil des années, d'un siècle à l'autre, aura donc fleuri une pluralité de lectures d'Au coeur des ténèbres, de l'exotique racisant au politique anticolonialiste, jusqu'au métaphysique, en passant par le psychologique s'appuyant sur l'état dépressif de Joseph Conrad.

Cette pluralité d'analyses contradictoires, en tout cas animée par des sentiments divers, sourd à travers la tonalité volontiers virulente ou radicale de certains commentaires dont ce dossier donne un aperçu. Qu'on voie uniquement en Conrad le parangon de la théorie du racisme florissant en Europe à l'époque (Blaise Ndala), ou qu'on lui attribue la paternité de la construction d'une « rhétorique de l'horreur » s'agissant du Congo, de l'Afrique centrale et des Grands Lacs (Frits Andersen, «Virunga Park, DR Congo. The heart of darkness of UNESCO World heritage »), la charge polémique d'Au cœur des ténèbres est incontestable (quelles qu'en aient été les motivations de son auteur) et c'est cette complexité que nous souhaitions mettre en lumière.

Il s'agissait en effet de questionner non seulement le caractère problématique de l'extraordinaire écho de ce texte via le placage simpliste de cette image puissamment évocatrice de "cœur des ténèbres" incessamment convoquée dès lors qu'il est question de discours sur l'Afrique; mais aussi, le caractère non moins problématique d'une réception qui pourrait bien elle-même se laisser prendre à son propre piège en renonçant à analyser méthodiquement les effets et les enjeux d'une exploitation systématique de ce texte littéraire consacré dans la construction d'un discours sur l'Afrique.

En fin de compte, on peut se demander si la réception de la novella «africaine " de Conrad ne repose pas sur un malentendu. Si, à travers ces différentes et parfois contradictoires lectures, ce texte, comme tout grand texte littéraire, du Moby Dick de Melville à La Métamorphose de Kafka, ne révélait pas simplement son caractère polysémique. Ou si, plutôt, soit en même temps, soit après toute lecture, il ne s'agit pas de revenir à ce qui a généré les lectures: la richesse structurelle de l'œuvre. Ce qu'entreprend Marc Porée dans «La fabrique d'un fleuve » où il se penche "d'aussi près que possible sur l'établi de l'écrivain, où se "fabrique" un fleuve de mots, de phrases, à la recherche de ce qu'il charrie en termes de souvenirs, de représentations, d'images, mais encore d'“agencements" ». Une approche où s'enchâssent les mots et leurs représentations, non seulement à l'intérieur du texte de la novella, mais aussi et plus largement dans l'œuvre entière de l'écrivain. Le lit, bed, n'est plus seulement celui du fleuve, mais où sont charriées, embarquées et emportées, les images, d'un texte, d'une époque, d'un lieu à l'autre, se répondant en des reflets multiples. Des reflets multiples où finissent peut-être par se perdre l'objet central, le fleuve, en une 
navigation à l'aveugle jusqu'à disparaître ou éclater dans une impasse de la construction, voire de la représentation. Et laisser, dès lors, à jamais place au débordement des interprétations et à leur dérive. Avec le vide, un blanc au centre.

Impasse représentative de l'aporie créative, presque substantielle, de l'auteur et artiste, impuissant à saisir l'objet de sa quête, et ne pouvant que l'approcher et le décrire par la tangente, "au-delà des faits réels", par touches impressionnistes, "tonalité » ou «vibration ». En se jouant « un petit peu (juste un petit peu)» du lecteur, pour avoir lui-même perdu au jeu, tout comme ses personnages. Laissant les "curieux" qu'analystes fourvoyés que nous sommes, "fureter dans toutes sortes d'endroits (où [nous n'avons] rien à faire pour en ressortir avec toute sorte de butin ${ }^{22}$. " Vain butin.

Pour conclure sans fermer et répondre à Boniface Mongo Mboussa ${ }^{23}$, ce n'est peut-être ni l'auteur qui gagne, ni ses épigones, c'est le texte. Le texte, telle la mer où dé-bouche le fleuve, toujours recommencé. En ses lectures. Un fleuve jamais identique et à nul autre pareil.

\section{NOTES}

1. Heart of Darkness, publié d'abord en trois parties dans Blackwood's Magazine en 1899, puis en 1902 chez William Blackwood dans un recueil de trois récits, Youth: a Narrative and Two Other Stories.

2. Voir Continents Manuscrits, $n^{\circ}$ 4, 2015, https://journals.openedition.org/coma/507

3. Royaume couvrant le nord de l'Angola actuel, l'enclave de Cabinda et une partie des territoires des Congo-Brazzaville et Congo-Kinshasa d'aujourd'hui. Né au XIII ${ }^{\mathrm{e}}$ siècle, il connut son apogée au $\mathrm{XV}^{\mathrm{e}}$ et $\mathrm{XVI}^{\mathrm{e}}$ siècles.

4. « My task is [...] before all, to make you see. That - and no more, and it is everything. [...] It is to show its vibration, its colour, its form ; and through its movement, its form, and its colour, reveal the substance of its truth ", Préface à The Nigger of Narcissus (Le Nègre de Narcisse, 1897).

5. Entre autres, Through the Dark Continent, 1878 [À travers l'Afrique, s.d.] et In Darkest Africa, 1890 [Dans les ténèbres de l'Afrique, 1890].

6. Diplomate irlandais (1864-1916) auquel Mario Vargas Llosa a consacré un roman, Le Rêve du Celte (Paris, Gallimard, 2011). Casement et Conrad se seraient rencontrés au Congo en 1890.

7. Mark Twain, Le Soliloque du roi Léopold, Bruxelles, Jacques Antoine éd., 1987, rééd. Paris, L'Harmattan, 2004 et 2008.

8. Conan Doyle, Le Crime du Congo belge, Paris, Les Nuits rouges, 2005.

9. «C'était un autre monde, et les hommes étaient - non, ils n'étaient pas inhumains. Eh bien voyez-vous, c'était ça le pire - se douter qu'ils n'étaient pas inhumains. [...] ce qui vous faisait frémir, c'était l'idée de leur humanité - semblable à la vôtre - la pensée de votre lointaine parenté avec ce tumulte effréné et passionné. » Joseph Conrad, Au cœur des ténèbres et autres récits, Paris, Gallimard, coll. « La Pléiade », 2017, p. 676.

10. «La remontée de ce fleuve, c'était comme une remontée aux premiers commencements du monde [...] ", Au cœur des ténèbres et autres récits, op. cit., p. 672.

11. Op. cit. 
12. «Piniau also suggests that anthologies and reference works, favor exotic passages (danger, combat, difficulties and savagery), but leave out the 'antiexotic' passages although they are more abundant and more characteristic (...) and everything Piniau refers to as 'realistic knowledge' (savoir réaliste) », Pierre Halen, "Stanley \& Conrad: Founders of Alternatives Discourses about Africa ", dans Elisabeth Mudimbe-Boyi éd., Remembering Africa, Portsmouth (NH), Heinemann, series "Studies in African literature", 2002, 339 p., p. 56-72, p. 67.

13. "En marge d'“Au cœur des ténèbres" ", Au cœur des ténèbres et autres récits, op. cit., p. 734.

14. Nicolas Martin-Granel, «Terrains d'Afrique ou l'auteur comme anthropologue », Études littéraires africaines, $\mathrm{n}^{\circ}$ 35, 2013, p. 144.

15. Yannick Haenel est, par ailleurs, l'auteur d'un article dans lequel il propose une lecture d'Au cœur des ténèbres à la fois profondément politique ( l'extermination est le secret des Temps modernes, et Kurtz en est le témoin ») et existentielle, voire métaphysique (" les “ténèbres”, c'est ce que Lacan nommait le réel - c'est-à-dire ce qui se dérobe à la prise, le trou qui rend impossible la représentation et fait vaciller tout rapport avec l'existence ») ou initiatique, celle d'une démarche sacrificielle ( "J'aime lire Au cœur des ténèbres, par-delà sa dimension politique, comme une descente dans les arcanes du sacrificiel. "J'ai assisté au mystère inconcevable d'une âme”, dit Marlow»), «Ce qui me brûle en lisant Conrad», En attendant Nadeau, n 44, https://www.enattendant-nadeau.fr/numero-44-conrad/

16. Dans sa préface au volume de La Pléiade (Au cœur des ténèbres et autres récits, op. cit.), Marc Porée cite aussi, dans le cadre de la Nouvelle-Guinée, Hors du charnier natal, biographie romancée de Christophe Claro (Paris, Inculte, 2016).

17. Pierre Halen, «Stanley \& Conrad: Founders of Alternatives Discourses about Africa », dans Elisabeth Mudimbe-Boyi éd., Remembering Africa, op. cit., p. 62. Plus loin, il complète sa definition : « The Other is not precisely the African in whom nobody is interested, especially since he might remind the reader of humanitarian problems or of its historical responsibility in regards to him. [...] But the Other is rather an object in whom the Westerner does not (want to) see himself or anything equivalent to himself [...] Thus, the Other is merely the figurative opposite to the historical subject: an extra on the screen, or something dubious to fight ", Pierre Halen, id., p. 62-65.

18. «[...] not only historians like Hochschild, but also vast numbers of international critics and literary researchers use Joseph Conrad in a faulty and twisted manner, resulting in significant historical blind spots": Frits Andersen, The Dark Continent? Images of Africa in European Narratives about the Congo ", Aarhus, Aarhus University Press, 2016, 690 p., p. 336.

19. Thomas Mpoyi-Buatu, La Re-Production, Paris, L'Harmattan, coll. « Encres noires, 1986.

20. Le Rwenzori est le nom d'une chaîne de montagnes située de part et d'autre de la frontière ougando-congolaise, dans le Rwenzori Mountains National Park en Ouganda et dans le Parc national des Virunga en RD Congo. Le Rwenzori est ainsi pris comme synecdoque de la province du Nord-Kivu.

21. C'est à la représentante spéciale de l'ONU pour la violence sexuelle dans les conflits, Margot Wallström, que l'on doit ce malheureux qualificatif, désormais devenu le stigmate de toute une région (l'Est du Congo) depuis près d'une décennie.

22. Toutes les citations de ce paragraphe proviennent de : Conrad, «En marge d'“Au cœur des ténèbres" ", op. cit., p. 734.

23. Voir la discussion à la suite de Blaise Ndala, «Au cœur des ténèbres, une œuvre ambiguë ». 
INDEX

Mots-clés : Conrad, Congo, Fleuve, Au cœur des ténèbres

\section{AUTEURS}

\section{JEAN-PIERRE ORBAN}

JEAN-PIERRE ORBAN est chercheur associé à l'Institut des textes et manuscrits modernes (CNRSENS, Paris), spécialisé en littérature francophone (Yambo Ouologuem, Paul Lomami Tchibamba, rapports auteur-éditeur entre centre et périphérie). Il dirige à l'ITEM le groupe de travail Schwarz-Bart. Écrivain, il est auteur de romans, de nouvelles et de pièces de théâtre.

\section{MAËLINE LE LAY}

MAËLINE LE LAY est chargée de recherche au CNRS, affectée à l'IFRA-Nairobi (Institut français de recherche en Afrique). Ses recherches portent sur le théâtre, les arts de la scène et la littérature en République Démocratique du Congo et dans la région des Grands Lacs. Elle a publié en 2014 chez Honoré Champion La parole construit le pays. Théâtre, langues et didactisme au Katanga. Elle a également co-dirigé, avec Dominique Malaquais et Nadine Siegert, Archive (re)mix. Vues d'Afrique (Presses universitaires de Rennes, 2015). 